\title{
An iatrogenic septic tenosynovitis of the digital flexor tendon sheath after diagnostic anaesthesia in a horse
}

\author{
Margriet C. Visser-Meijer ${ }^{7}$ and Astrid B. M. Rijkenhuizen² \\ Veterinary Equine Clinic, Honselersdijk and Veterinary Clinic Duurstede, Wiik bij Duurstede², The Netherlands
}

\begin{abstract}
Summary
This report describes the unsuccessful treatment of an iatrogenic septic tenosynovitis following diagnostic anaesthesia of the digital flexor tendon sheath in an 11 -year-old Dutch Warmblood gelding. Diagnostic anaesthesia is a valuable tool in localising the cause of lameness, but is not without risk. After diagnosing the septic tenosynovitis, the horse was treated with local and systemic antibiotics, nonsteroidal antiinflammatory drugs and was subjected to a total of three tenoscopies in conjunction with copious lavage of the deep digital tendon sheath. During the second tenoscopy a desmotomy of the palmar annular ligament was performed to relieve constriction of the tendons. After the first two tenoscopies the surgical portals were closed. After the third tenoscopy the surgical incisions were left open for open drainage. Because of the development of a non-weight-bearing lameness after a long and aggressive treatment period the horse had to be euthanized and was cremated. The septic tenosynovitis was considered to be of iatrogenic origin, as a complication of the diagnostic anaesthesia during the lameness examination.
\end{abstract}

Keywords: horse / iatrogenic / septic tenosynovitis / digital flexor tendon sheath / diagnostic anaesthesia

\begin{abstract}
latrogene septische Entzündung der distalen gemeinsamen Beugesehnenscheide nach diagnostischer Anästhesie bei einem Pferd

Die Diagnostische Anästhesie ist eine wertvolle Hilfe bei der Lokalisierung von Lahmheiten, sie ist jedoch nicht ohne Risiko. Ein 11 -jähriger Holländer-Wallach erlitt nach einer diagnostischen Anästhesie der distalen gemeinsamen Beugesehnenscheide eine septische Tendovaginitis. Nachdem die septische Tendovaginitis diagnoistiziert wurde das Pferd systemisch und lokal mit Antibiotika sowie mit nichtsteroidalen Antiphlogistika behandelt. Im Laufe von drei Tenoskopien wurde die Sehnenscheide intensiv gespült. Anlässlich der zweiten Tenoskopie wurde das Ringband durchtrennt, um die eingetretene Konstriktion der Sehnen zu vermindern. Nach den beiden ersten Tenoskopien waren die chirurgischen Zugänge verschlossen worden, nach der letzten Tenoskopie wurde der Zugang zum Zweck der Drainage offen gelassen. Nachdem das Pferd nach langdavernder und aggressiver Behandlung eine starke Lahmheit entwickelte und die betroffene Gliedmaße nicht mehr belastete, wurde es euthanasiert und kremiert. Die septische Sehnenscheidenentzündung wurde als iatrogenen Ursprungs betrachtet, verursacht durch die diagnostische Anästhesie.
\end{abstract}

Schlüsselwörter: Pferd / iatrogen / septisch / Sehnenscheidenentzündung / Beugesehnenscheide / diagnostische Anästhesie / Orthopädie

\section{Introduction}

Despite the many technological advances in equine sports medicine over the past decades, diagnosing lameness is still a challenge. A diagnosis is usually derived from the results of the clinical examination, anatomical knowledge and additional diagnostic procedures (Ross et al. 2003). Diagnostic anaesthesia is valuable tool in localising the cause of lameness (Rijkenhuizen 2001, Ross et al. 2003, Riggs 2013). When lameness diminishes, disappears, or switches to the other leg after a diagnostic anaesthesia, the source of pain is localised within the anaesthetized region. However, knowledge of false negative as well as false positive responses to diagnostic anaesthesia is essential in the clinical search for the cause of lameness (Rijkenhuizen 2001, Ross et al. 2003, Riggs 2013). Furthermore, diagnostic anaesthesias are not without risk, and minor local inflammatory reactions after perineural anaesthesia occur (Ross et al. 2003). Also iatrogenic septic synovitis after intrasynovial injections has been described (Schneider et al. 1992a, Meijer et al. 2000, Ross et al. 2003, Bergman 2010, Smith 2010).

This case report describes an iatrogenic septic tenosynovitis of the digital flexor tendon sheath after perineural diagnostic anaesthesia in a horse, which, as far as the authors are aware has not been reported in the literature thus far.

\section{Case history and clinical findings}

An 11 -year-old Dutch Warmblood gelding actively competing at Prix St George level dressage was presented to the clinic with a lameness for two weeks. The slight lameness had an acute onset but the horse was continued to be worked. The horse showed a grade 1 (AAEP scale 1-5) right forelimb (RF) lameness during trotting on a straight line. The clinical examination revealed no detectable abnormalities or painful responses after palpation of both forelimbs, with the exception of a slight distension of the coffin joint region (coronary band) at both forelimbs. The flexion test of the RF metacarpophalangeal joint was slightly positive with a grade 1 increase over the original lameness. The flexion test of the left metacarpophalangeal joint was negative.

The lateral and medial palmar digital nerves of the RF were anesthetised as distal as possible in the pastern region with $20 \mathrm{mg}$ of lidocaine hydrochloride $\left(1 \mathrm{ml}\right.$ Lidocaine $\mathrm{HCL} 2{ }^{\circledR}{ }^{\circledR}$, Eurovet Animal Health BV) at each site. No gait change on a 
straight line could be observed. After a lateral and medial palmar digital nerve block at the base of the proximal sesamoid bones (abaxial sesamoid block) using $40 \mathrm{mg}$ of lidocaine hydrochloride $\left(2 \mathrm{ml}\right.$ Lidocaine $\mathrm{HCL} 2 \%{ }^{\circledR}$, Eurovet Animal Health BV) at each site the horse became sound in ten minutes.

Radiographs of the distal phalanges, the navicular bone and the metacarpophalangeal joint of the RF (lateromedial, dorsoproximal-palmarodistal oblique and dorsopalmar views of the distal phalanx placed in a firm block with an angle $45^{\circ}$ and the dorsopalmar, lateromedial, dorsolateral-palmaromedial and dorsomedial-palmarolateral views of the metacarpophalangeal joint) revealed no radiographic abnormalities. Because no radiographic changes were observed and the lameness was only located in the RF, no radiographs were taken from the LF.

A distal interphalangeal joint problem was suspected and because of the short duration as well as the low-grade of the lameness and the horse's sport expectations in the short term, it was decided to treat the distal interphalangeal joint. After shaving, scrubbing the site of injection with chloorhexidine (Hibiscrub ${ }^{\circledR}$, Regent Medical Overseas Ltd.) and desinfecting it with isopropyl alcohol (Sterillium ${ }^{\circledR}$, Hartmann Group), a combination of $20 \mathrm{mg}$ of sodium hyaluronate $\left(2 \mathrm{ml} \mathrm{Hylartil}{ }^{\circledR}\right.$ Vet. Sterile solution, Pfizer Animal Health BV) and $10 \mathrm{mg}$ of triamcinolonacetonide (1 ml Depocort ${ }^{\circledR}$, Ceva Sante Animale BV) was injected in the dorsal pouch of the joint lateral to the common digital extensor tendon. The synovial fluid that was aspirated prior to injection of the medications was clear and viscous. The owners were advised to handwalk the horse for four days and start training afterwards.

The next day the horse developed a severe (grade 4/5) RF lameness and was referred to the clinic again. The body temperature was $37.4^{\circ}$ Celsius. No heat or swelling of the distal interphalangeal joint was detected. Passive flexion of the distal foot revealed no reaction. Arthrocentesis of the distal interphalangeal joint revealed clear and viscous synovial fluid, which was discarded. To exclude fractures of the distal phalanges dorsopalmar, dorsolateral-palmaromedial oblique and dorsomedial-palmarolateral oblique radiographs of the distal RF were taken. Because no fractures could be identified, a post-injection flare of the distal interphalangeal joint was suspected as the cause of acute lameness. The horse was kept in the hospital and a non-steroidal-anti-inflammatorydrug (flunixin meglumin 1,0 mg/kg BW three times daily (Meflosyl ${ }^{\circledR}$, Forte Dodge Animal Health Benelux BV)) was given intravenously. Besides, it was decided to treat the distal interphalangeal joint intraarticularly after aseptic preparation with $10 \mathrm{mg}$ of gentamycin sulphate (Genta-ject 10\% ${ }^{\circledR}$, Dopharma BV) and administer antibiotics intravenously (sodium ampicillin 10mg/kg BW twice daily (Ampi-Dry 5000 ${ }^{\circledR}$, Dopharma BV) and gentamicin sulphate $6,6 \mathrm{mg} / \mathrm{kg}$ BW once daily (Gentaject 10\% ${ }^{\circledR}$, Dopharma BV)).

The next day the horse showed good improvement from a grade 4 to 1 lameness and this did not change until the third day. At that moment a grade 2/5 lameness of the RF was noticed, which worsened to non-weight-bearing lameness by the fourth day. A marked distension of the digital flexor tendon sheath (DFTS) proximal of the palmar annular ligament was recognised. The distension was warm and painful at palpation.

Synoviocentesis of the DFTS yielded a large quantity of cloudy turbid synovial fluid. Cytological evaluation of the synovial fluid revealed a septic synovitis of the DFTS with an increased white blood cell (WBC) count of 35,5 G/l with 91,5\% of neutrophils. No sample was submitted for microbial culture and antimicrobial sensitivity. It was decided to subject the horse immediately to a tenoscopic surgery under general anaesthesia. In left lateral recumbency the tendon sheath of the RF was prepared for aseptic surgery. The sheath was distended with $\mathrm{NaCl}$ 0,9\% solution and the arthroscope was placed in the lateral pouch of the DFTS between the palmar annular ligament (PAL) and the proximal digital annular ligament as described by Nixon (1990). While evaluating the tendon sheath by flexing the metacarpophalangeal joint, a reddish synovium with some fibrin in the lateral and medial pouches and no abnormalities of the deep and superficial flexor tendon were observed. An instrument portal was made in the lateral pouch of DFTS just proximal of the PAL for debridement of the fibrin and a thorough lavage with $6 \mathrm{~L}$ of $\mathrm{NaCl}$ $0,9 \%$ solution. At the end of the surgery just before suturing the tenoscopic portals $10 \mathrm{mg}$ of gentamicin sulphate was administered intrathecally. The intravenous antibiotic and anti-inflammatory treatment was continued as prior to surgery. Already the next day the horse was weight bearing the RF and showed a grade 1 lameness.

After 3 days a decline was observed again and lameness increased from grade 1 to 3 . Synoviocentesis of the DFTS revealed a turbid synovial fluid with an elevated WBC count of 38,3 G/l and 93,6\% of neutrophils. Again a tenoscopy of the DFTS, as described before, was performed under general anaesthesia. The arthroscope was introduced into the sheath through the same incision. The synovium was reddish and oedematous swollen, a lot of fibrin deposits were detected inside the sheath and again both tendons did not show any abnormalities. A desmotomy of the PAL was performed using a hooked knife followed by a debridement of the fibrin deposits and a thorough lavage of the DFTS with $6 \mathrm{~L}$ of $\mathrm{NaCl}$ $0,9 \%$ solution. A synovial sample was submitted for bacterial culture and its antimicrobial sensitivity. The antibiotic treatment was changed to cefquinome (1 mg/ $\mathrm{kg}$ BW once daily (Cobactan ${ }^{\circledR}$, Intervet Schering Plough Animal Health) intramuscularly). The anti-inflammatory treatment was continued with flunixin meglumin $(1,0 \mathrm{mg} / \mathrm{kg}$ BW three times daily (Meflosyl ${ }^{\circledR}$, Forte Dodge Animal Health Benelux BV) intravenously). Again, the lameness diminished remarkably after the tenoscopy of the DFTS to a grade 1 lameness.

The culture of the synovial fluid sample revealed a Methicillin-Resistent-Staphylococcus-Aureus (MRSA), which was only sensitive for trimethoprim-sulfamethoxazol. The antibiotic regime was changed to trimethoprim-sulfadiazine immediately $(5 \mathrm{mg}$ trimethoprim and $25 \mathrm{mg}$ sulfadiazine twice daily (Sulfatrim ${ }^{\circledR} 400 \mathrm{mg} / \mathrm{g}$ Oral Paste, AST Farma BV) orally). The surgical wounds closed primarily in nine days. The anti-inflammatory treatment was continued for one week after the second lavage.

During its stay at the clinic the horse was kept at box rest and showed no body temperature above $38.0^{\circ}$ Celsius. Three 
weeks after the second tenoscopy, ultrasonography of the DFTS and tendons was performed with a linear-array 10.0 $\mathrm{MHz}$ transducer (MyLab Ô 50X Vision from Esaote Benelux Bv). After shaving the region of the DFTS from halfway the third metacarpal bone distally to the hoof and wetting the skin, ultrasonography was performed without a pad on a loaded leg. A prominent fluid distension and a thickened tendon sheath wall were visible. The PAL was not thickened $(1,4 \mathrm{~mm})$ and a longitudinal tear in the medial part of the deep flexor tendon at the height of the proximal digital annular ligament was suspected. Although the clinical situation of the horse was stable at that moment, it was decided on base of a septic condition in the DFTS and the tear in de deep flexor tendon to continue the antibacterial treatment for one month. Two days after finishing the antibacterial treatment, the horse was released from the clinic. Four weeks box rest were advised. At the moment of discharge the horse still showed a grade 1 lameness.

A week later, the horse was readmitted suffering a nonweight-bearing lameness of its RF. For the first time the horse showed an elevated body temperature of $38,5^{\circ}$ Celsius. The distal limb from halfway the metacarpal bone to the hoof was oedematous, thickened, warm and painful, especially at palpation of the pouches of the DFTS. Synoviocentesis of the DFTS revealed an increased amount of turbid synovial fluid with a markedly elevated WBC count of $52,5 \mathrm{G} / \mathrm{l}$ and $95,5 \%$ of neutrophils. A tenoscopic assisted debridement and lavage ( $9 \mathrm{~L}$ of $\mathrm{NaCl} 0,9 \%$ solution) in left lateral recumbency under general anaesthesia was performed, using a $5 \mathrm{~mm}$ arthroscopic portal palmar to the original portal and an instrumental in the lateral pouch of DFTS just proximal of the PAL. Distending the DFTS with $0,9 \% \mathrm{NaCl}$ solution was not necessarily, because the sheath itself was already very distended. Inside the tendon sheath large fibrin clots and voluminous fibrous adhesions were present in all its pouches. After some flushing to clear up the inside of the sheath, a longitudinal tear at the medial border of the deep flexor tendon running from under the PAL until the distal digital annular ligament was detected and treated by shaving the torn fibrils with the help of an electrical shaver (FMS Duo ${ }^{\circledR}+$ system from FMS group). Afterwards, the tenoscopic portals were left open to allow passive drainage. The portals were covered with a sterile pad (Medicomp ${ }^{\circledR}$, Hartmann Group) and a sterile bandage of cotton was applied. The sterile bandage was changed every day after the skin around the tenoscopic incisions was cleaned with povidone-iodine soap and disinfected with isopropyl alcohol (Sterilium ${ }^{\circledR}$, Hartmann Group). Treatment with trimethoprim-sulfadiazine $(5 \mathrm{mg}$ trimethoprim and $25 \mathrm{mg}$ sulfadiazine twice daily (Sulfatrim ${ }^{\circledR}$ $400 \mathrm{mg} / \mathrm{g}$ Oral Paste, AST Farma BV) orally) was re-initiated prior to surgery. Additionally, phenylbutazone $(2 \mathrm{mg} / \mathrm{kg} \mathrm{BW}$ twice daily (Equipalazone ${ }^{\circledR}$, Dechra) orally) was administered. After one week the horse improved and lameness slightly diminished to a grade 3. However, the tenoscopic portals still drained a purulent exudate and the leg was very oedematous. After 10 days, the horse was non-weight-bearing again. Radiography of the DFTS region (a dorsopalmar, a lateromedial, a dorsolateral-palmaromedial and a dorsomedial-palmarolateral view) revealed that all bony structures showed osteoporotic decalcification. Ultrasonography of the DFTS and tendons was performed as described before except on a non-weight-bearing leg. The proximal part of the superficial flexor tendon proximal from the palmar annular ligament showed a normal to hyperechogenic structure, an increased diameter with an irregular contour. Because of the non-weight-bearing situation the other structures could not be evaluated reliably. In consultation with the owner, it was decided to euthanize the horse because of the persistent massive infection of the DFTS, resistant to aggressive treatment. The horse had to be cremated and no post-mortem examination was available.

\section{Discussion}

Although lameness examinations are routinely performed in daily equine practice, complications can occur. They can vary from meaningless to more serious and even life threatening. The same is true for the use of diagnostic injections. They are not without risk, although the complication rate after intraarticular injection of 0,14\% can be considered as low (Bergman 2010). The local anaesthetics most frequently used for diagnostic injections are 2\% lidocaine hydrochloride and 2\% mepivacaine hydrochloride. These solutions are potent and rapidly effective, but also irritating (Ross at al. 2003). For both perineural blocks and intrasynovial injections, mepivacaine is less irritating than lidocaine (Hall et al, 1991, Ross et al. 2003) and in all cases, the least amount of local anaesthetic should be used, because of the residual tissue irritation (Stashak 1987). In the presented case lidocaine was used, simply because mepivacaine is not licensed for its use in horses in The Netherlands. Additionally, the authors experienced no prior irritating reactions in horses after local anaesthesia with lidocaine.

Acute cellulitis after extrasynovial injection (Goldfarb et al. 2007) or acute synovitis after intrasynovial injection (most commonly intra-articular), a so-called flare, are rare complications in a lameness examination (Kuemmerle et al. 2006, Smith 2010). A flare may occur following injection of many substances, including local anaesthetics, HA, corticosteroids or PSGAGs (Cole et al. 2005, Kuemmerle et al. 2006, Smith 2010). Such reactions are much less common with the use of local anaesthetics than other medications (Ross et al. 2003). However, it is one of the most difficult conditions to differentiate from a synovial infection (Kuemmerle et al. 2006), which itself is also a possible sequela of a lameness examination when an intrasynovial anaesthesia has been performed (Schneider et al. 1992a, Meijer et al. 2000, Ross et al. 2003, Bergman 2010). The clinical signs are similar (severe lameness, joint distension and an acute inflammation inside the joint) and there are no easy means of differentiation. It is suggested, that nucleated cell counts are often lower with a flare than with a synovial infection and that total protein is high with a flare. Early onset of clinical signs $(<12 \mathrm{~h})$ is suggestive of a flare and horses with a flare usually respond well to a single dose of NSAIDs where after the clinical signs gradually resolve over 48-72 h (Smith 2010).

In the present case, two perineural blocks were performed in the distal forelimb. After completing the lameness examination an intrasynovial injection of the distal interphalangeal joint was performed with a combination of hyaluronic acid and corticosteroids. However, the arthrocentesis was performed distant from the local anaesthesia sites. Within 24 hours, 
an acute severe lameness developed. Because of the acute onset, no other clinical relevant findings and the normal appearance of the synovial fluid a postmedication flare of the distal interphalangeal joint was suspected and treatment with NSAIDs and antibiotics was initiated. The antibiotics were applied because a septic arthritis could not be excluded as a dose of triamcinolonacetonide was added to the injection at the time of treatment.

Four days post treatment the horse became non-weight-bearing at the RF and a septic tenosynovitis of the DFTS was diagnosed. A septic tenosynovitis is a common problem in horses. It usually occurs secondary to direct bacterial contamination of the sheath by a wound, surgery or an intrasynovial injection (Schneider et al. 1992a). Haematogenous invasion of bacteria into a tendon sheath is frequently seen in foals, but has rarely been reported in adult horses (Honnas et al. 1991, Schneider et al. 1992a).

In the present case, surgery and a haematogenous invasion were not considered as likely causes of the septic condition of the DFTS. An invisible puncture wound could not be excluded. The aetiology of the infection has a significant effect on the type of bacteria identified by culture (Schneider et al. 1992a). Horses that develop an infection secondary to a penetrating wound frequently provide cultures of more than one organism (Schneider et al. 1992a, Meijer et al. 2000, Taylor et al. 2010). In this case, only one organism, a Methicillin-ResistentStaphylococcus-Aureus (MRSA) was cultured from the DFTS. The most common infective organism resulting from synoviocentesis is Staphylococcus-Aureus (Schneider et al. 1992a, Meijer et al. 2000, Taylor et al. 2010). Whereas only perineural blocks were performed, an intrasynovial injection was not primary thought to be the cause of the septic tenosynovitis. However, the lateral and medial palmar digital nerves are located just palmar to their respective artery and vein and lie along the dorsal border of the DFTS. Anatomically, there is the risk of puncturing the DFTS while blocking the lateral and medial palmar digital nerves (Rijkenhuizen 2001).

Conclusively, the septic tenosynovitis in this case report is considered iatrogenic, caused by the perineural blocks of the palmar digital nerves in the distal forelimb. Distal perineural blocks are routinely used in equine practice and a septic tenosynovitis after such anaesthesias has a very low prevalence. Therefore a septic tenosynovitis can be considered as an extremely rare complication of perineural blocks. Because of the rareness of this complication a septic tenosynovitis was not primary considered as the cause of the lameness. The more logical cause "flare" was initially diagnosed, but deemed to be false, because the synovial fluid withdrawn form the treated joint prior to the repeat-injection of antibiotics did not reveal any abnormality. The quick onset of the nonweight-bearing lameness, not performing a synoviocentesis of the DFTS during the initial lameness examination, no distension of the DFTS on the first day of the non-weight-bearing lameness and no reaction to the passive flexion of the distal limb at that time were misleading circumstances. The initial clinical improvement after starting treatment seemed to justify the presumed diagnosis of a flare.

However, it cannot be emphasized enough, that knowledge of the direct outcomes and effects after diagnostic anaesthe- sia have to be thoroughly understood for interpreting symptoms during and after lameness examinations. Furthermore, infectious synovitis should always be considered in the differential diagnosis when a severe lameness of acute onset is present (Stover 1990)

Failure to culture bacteria from synovial fluid obtained of joints, bursae or sheaths that are suspected of being infected is common (Schneider et al. 1992a, Meijer et al. 2000). Contributing factors include incorrect handling of samples, inadequate laboratory technique, the intrinsic bactericidal properties of septic synovial effusions, the absence of the bacteria in the synovial fluid itself and a previous antibiotic treatment (Stashak 1987, Honnas et al. 1991, Schneider 1992a). In this case at the moment of the first synoviocenthesis of the DFTS, the horse had already been treated with antibiotics for four days and it was therefore decided not to culture the synovial fluid. However, culturing a MRSA and its antimicrobial sensitivity in the synovial fluid after the second tenoscopy proves that culturing is worthwhile. It should have been performed with the synovial fluid from the first synoviocenthesis of the DFTS and also with the synovial fluid from the synoviocenthesis of the coffin joint, although a flare in this this joint was suspected. An infected coffin joint was not already excluded at that time. This would have triggered the required change of antibiotics to trimethoprim-sulfadiazine earlier. Regional intravenous administration of antimicrobial drugs was not applied in this case and might have been a good option (Wright et al. 2003). However, the authors do not commonly use this technique for treating a septic synovitis, based on their experiences described earlier (Meijer et al. 2000)

Equally important as the antimicrobial treatment is drainage of the infected tendon sheath. Synovial lavage is meant to relieve intrathecal pressure, to decrease the number of microorganisms at the site of infection, to remove cellular debris and devitalised material, inflammatory exudate and fibrin and enhance the environment (optimise the $\mathrm{pH}$ and remove antimicrobial inhibitors) for the activity of antimicrobial drugs (Stover 1990, Honnas et al. 1991, Meijer et al. 2000, Wright et al. 2003, Wereszka et al. 2007). According to the authors a repeated through-and-through lavage every other day in treating septic arthritis until the white cell count drops below $15 \mathrm{G} / \mathrm{l}$ reveals a good overall joint recovery rate of $81 \%$ in adult horses (Meijer et al. 2000). In the present case a tenoscopic assisted lavage resulted in an initial improvement of the horse. Because lavage and debridement under endoscopic view of septic synovitis in the horse has been described as being successful, it was decided to treat this tenosynovitis as described (ter Braake 1992, Wright et al. 2003, Wereszka et al. 2007). Endoscopic treatment of infected synovial cavities permits thorough evaluation with appropriate debridement, effective lavage and minimal tissue trauma (Wright et al. 2003). However, based on high survival rates documented with aggressive treatments in eliminating infection in adult horses treated for septic arthritis/tenosynovitis (Schneider et al. 1992a, Meijer et al. 2000), it could have been a good suggestion to combine the endoscopic surgery with controlling the synovial WBC count every other day in this case and maybe recurrence could have been prevented.

At the third readmittance, it was decided to facilitate open drainage after the tenoscopy to eliminate the synovial infec- 
tion (Schneider et al. 1992b, Chan et al. 2000, McNally et al. 2013). Open drainage has been described as an effective method of achieving chronic drainage and is indicated in horses that have established chronic intrasynovial infections or that do not respond to joint/sheath lavage (Schneider et al. 1992b, McNally et al. 2013)

Septic tenosynovitis is a serious problem in the horse with a guarded to poor prognosis for return to their previous level of athletic function (Honnas et al. 1991, Smith et al. 2006, Wereszka et al. 2007). The prognosis even declines for horses with a positive bacterial culture from a septic synovitis, as it was in this case (Taylor et al. 2010). An early diagnosis is paramount to a successful treatment of infected synovial structures (Stover. 1990, Honnas et al. 1991). The prognosis for horses with a septic tenosynovitis is documented to improve if treatment is initiated within 24 hours of injury (Wereszka et al. 2007). The failure of diagnosing the septic tenosynovitis of the DFTS at the first time of the severe lameness in this case caused a delay of four days in starting an effective treatment. This delay diminished the prognosis for this horse immediately.

It is suggested that the prognosis of a septic tenosynovitis will improve with a desmotomy of the palmar annular ligament to decompress the structures inside the tendon sheath and to facilitate the removal of fibrin in the different pouches (Honnas et al. 1991 and Chan et al. 2000). So, this was performed during the second tenoscopy. Horses with concurrent tendon injuries were less likely to return to their previous level of performance than horses without such injuries (Wereszka et al. 2007). In the presented case the horse developed a lesion of the deep digital flexor tendon, which could be treated during the third tenoscopy. However, later on there was the suspicion of an infected superficial flexor tendon, which validated the decision to euthanize the horse.

This case report makes it clear that in the beginning a rather innocent lameness examination can result in a life-threatening situation. Although intrasynovial blocks after perineural blocks might occur (Rijkenhuizen 2001, Ross et al. 2003), as far as the authors are aware no septic tenosynovitis or septic arthritis after perineural blocks have been reported in the literature thus far. This case report demonstrates that iatrogenic tenosynovitis of the DFTS can occur in the horse as a rare complication following a perineural block.

\section{References}

Bassage // L. H. and Ross M. W. (2003) Diagnostic Analgesia. In: Ross M.W. and Dyson S.J. eds. Diagnosis and management of lameness in the Horse. Elsevier Science: Missouri, USA, 93

Bergman M. (2010) Inquiry of the appearance of complications with joint punctures of the horse. Dissertation, University of Leipzig, Germany

ter Braake F. (2002) Direct endoscopic approach improves prognosis of septic synovitis in the horse. Tijdschr. Diergeneeskd. 127, 444

Chan C. C., Murphy H. and Munroe G. A. (2000) Treatment of chronic digital septic tenosynovitis in 12 horses by modified open annular ligament desmotomy and passive open drainage. Vet. Rec. 147,388
Cole B. J. and Schumacher H. R. Jr. (2005) Injectable corticosteroids in modern practice. J. Am. Acad. Orthop. Surg. 13, 37

Goldfarb C. A., Gelberman R. H., McKeon K., Chia B. and Boyer M. I. (2007) Extra-articular steroid injection: early patient response and the incidence of flare reaction. J. Hand. Surg. Am. 32, 1513

Hall L. W. and Clarke K. W. (1991) General principles of local analgesia. In: Hall L.W. and Clarke K.W. eds. Veterinary Anaesthesia. 9th ed. Baillière Tindall: London, 173

Honnas C. M., Schumacher L., Cohen N. D., Watkins J. P. and Taylor T. S. (1991) Septic tenosynovitis in horses: 25 cases (1983-1989). J. Am. Vet. Med. Assoc. 199, 1616

Kuemmerle J. M., Uhlig H. and Kofler J. (2006) Severe acute inflammatory reaction (SAIR) of the fetlock joint after intraarticular hyaluronate injection in a horse. Vet. Comp. Orthop. Traumatol. 19, 236

McNally T. P., Slone D. E., Hughes F. E. and Lynch T. M. (2013) Tenosynoviotomy for sepsis of the digital flexor tendon sheath in 9 horses. Vet. Surg. 41, 114

Meijer M. C., van Weeren P. R. and Rijkenhuizen A. B. (2000) Clinical experiences of treating septic arthritis in the equine by repeated joint lavage: a series of 39 cases. J. Vet. Med. Physiol. Pathol. Clin. Med. 47, 351

Nixon A. J. (1990) Endoscopy of the digital flexor tendon sheath in horses. Vet. Surg. 19, 266

Rijkenhuizen A. B. M. (2001) Die diagnostischen anästhesien an der vordergliedmaße des pferdes/Diagnostic anaesthesia in the front limb oft he horse. Pferdeheilkunde 17, 330

Riggs C. M. (2013) Diagnosis of lameness - keeping an open mind. Eq. Vet. Educ. 25, 229

Schneider R. K., Bramlage L. R., Moore R. M., Mecklenburg L. M., Kohn C. W. and Gabel A. A. (1992a) A retrospective study of 192 horses affected with septic arthritis/tenosynovitis. Equine Vet. J. 24, 436

Schneider R. K., Bramlage L. R., Mecklenburg L. M., Moore R. M. and Gabel A. A. (1992b) Open drainage, intra-articular and systemic antibiotics in the treatment of septic arthritis/tenosynovitis in horses. Eq. Vet. J. 24, 443

Smith L. J., Mellor D. J., Marr C. M. and Mair T. S. (2006) What is the likelihood that a horse treated for septic digital tenosynovitis will return to its previous level of athletic function? Equine Vet. J. 38, 337

Smith M. R. W. (2010) Equine synovial fluid: synoviocentesis and analysis. Proceedings of the 49th British Equine Veterinary Association Congress. Burmingham

Stashak T. S. (1987) Diagnosis of lameness. In: Stashak T.S. ed. Adams' Lameness in horses, 4th ed. Lea \& Febiger: Philadelphia, USA, 100

Stover S. M. (1990) Infectious arthritis and tenosynovitis in the horse. Proceedings of the 12th Bain-Fallon Memorial Lectures. Townsville

Taylor A. H., Mair T. S. A., Smith L. J. and Perkins J. D. (2010) Bacterial culture of septic synovial structures of horses: Does a positive bacterial culture influence prognosis? Equine Vet. J. 42, 213

Wereszka M. M., White /I N. A. and Furr M. O. (2007) Factors associated with outcome following treatment of horses with septic tenosynovitis: 51 cases (1986-2003). J. Am. Vet. Med. Assoc. 230, 1195

Wright I. M., Smith M. R. W., Humphrey D. J., Eaton-Evans T. C. J. and Hillyer M. H. (2003) Endoscopic surgery in the treatment of contaminated and infected cavities. Equine Vet. J. 35, 613

\author{
M.C. Visser-Meijer \\ Veterinary Centre, Equine department \\ Harteveldlaan 1 \\ 2675 LE Honselersdijk \\ The Netherlands \\ margrietvisser@xs4all.nl
}

